\title{
WEIGHTED NORM INEQUALITIES FOR MULTIPLIERS
}

\author{
CRISTIAN E. GUTIERREZ
}

(Communicated by Richard R. Goldberg)

ABstract. We consider the two-weight function problem for a class of multiplier operators that include the Riesz and Bessel potentials.

1. Let $\varphi(t)$ be a nonnegative function on $(0, \infty)$ such that

$$
m(\xi)=\int_{0}^{\infty} \varphi\left(t^{1 / 2}\right) e^{-t \xi} \frac{d t}{t}
$$

is finite for every $\xi>0$. We define the operator $T$ by

$$
(T f)^{\wedge}(x)=m\left(\pi|x|^{2}\right) \hat{f}(x)
$$

where $\hat{f}(x)=\int_{\mathbf{R}^{n}} f(y) e^{-2 \pi i x \cdot y} d y$. In this note we prove weighted norm inequalities for the class of multiplier operators defined by (1.1). This class includes the Riesz potentials and the Bessel potentials by taking $\varphi(t)=t^{\alpha}$ and $\varphi(t)=t^{\alpha} e^{-t^{2}}, \alpha>0$, respectively. The case of the Riesz potentials has been studied in [5] and [3].

A weight function $u$ is said to belong to $D_{\mu}, \mu \geq 1$, if $u\left(B_{t s}(x)\right) \leq c t^{n \mu} u\left(B_{s}(x)\right)$ for every $t>1, s>0$ and $x \in \mathbf{R}^{n}$, where $B_{s}(x)$ denotes the ball with center $x$ and radius $s$ and $u\left(B_{s}(x)\right)$ its $u$-measure. We write $D_{\infty}=\bigcup_{\mu \geq 1} D_{\mu}$. Analogously, $u \in R D_{\nu}, \nu>0$, if $u\left(B_{s t}(x)\right) \geq c t^{n \nu} u\left(B_{s}(x)\right)$ for every $t \geq 1, s>0$ and $x \in \mathbf{R}^{n}$. It is not hard to see that if $u \in D_{\infty}$ then $u \in R D_{\nu}$ for some $\nu>0$. $L_{u}^{p}$ is the class of functions $g$ such that $\|g\|_{p, u}=\left(\int_{\mathbf{R}^{n}}|g(x)|^{p} u(x) d x\right)^{1 / p}$ is finite. $S_{0,0}$ will denote the class of Schwartz functions whose Fourier transforms have compact support not including the origin. The space $H_{u}^{p}, 0<p<\infty$, consists of all tempered distributions $f$ such that $\|f\|_{H_{u}^{p}}=\|N(f)\|_{p, u}$ is finite, where $N(f)(x)=$ $\sup _{|x-y| \leq t}|F(y, t)|, F(x, t)=f * \Psi_{t}(x), \Psi_{t}(\cdot)=t^{-n} \Psi(\cdot / t), \Psi \in S$. If $u \in D_{\infty}$, $\|f\|_{H_{u}^{p}}$ is equivalent to $\left\|N_{0}(f)\right\|_{p, u}$, where $N_{0}(f)(x)=\sup _{t>0}|F(x, t)|$ (see [8]).

We prove the following

THEOREM 1. Let $0<p<q<\infty, u \in D_{\mu}, v \in D_{\infty} \cap R D_{\nu}$ with $\nu q>\mu p$. If

$$
\varphi(t) u\left(B_{t}(x)\right)^{1 / q} \leq c v\left(B_{t}(x)\right)^{1 / p}
$$

for every $t>0$ and every $x \in \mathbf{R}^{n}$ then

$$
\|T f\|_{q, u} \leq c\|f\|_{H_{v}^{p}} \quad \text { for every } f \in S_{0,0} .
$$

THEOREM 2. Let $0<q \leq 1$ and $u, v \in D_{\infty}$. If

$$
u(Q) \int_{0}^{l}\left(\int_{s}^{2 s} \varphi(t) \frac{d t}{t}\right)^{q} \frac{d s}{s} \leq c v(Q)
$$

Received by the editors August 23, 1986 and, in revised form, October 30, 1986.

1980 Mathematics Subject Classification (1985 Revision). Primary 42B15, 42B30, 42B25. 
for every cube $Q$ ( $l$ is the edgelength of $Q)$, then

$$
\|T f\|_{q, u} \leq c\|f\|_{H_{v}^{q}} \quad \text { for every } f \in S_{0,0} .
$$

If $1<p<\infty$ and $v$ is such that $\|\cdot\|_{p, v} \approx\|\cdot\|_{H_{v}^{p}}$, Theorem 1 gives results for $L^{p}$. (For such $v$ see [1].) The method used to prove our theorems is similar to that in [5] and [3]. The unweighted case has been considered in [6]. When $v \equiv 1$ and $p=q$ a characterization of $u$ in terms of a maximal function associated with the kernel of $T$ is given in [7].

2. We state two lemmas which will be needed later.

LEMMA (2.1). Let $\sigma$ be a measure on $\mathbf{R}_{+}^{n+1}$ and $v \in D_{\infty}$ on $\mathbf{R}^{n}$. Then for $0<p \leq q<\infty$,

$$
\left(\int_{\mathbf{R}_{+}^{n+1}}|F(x, t)|^{q} d \sigma(x, t)\right)^{1 / q} \leq c\left(\int_{\mathbf{R}^{n}} N(f)(x)^{p} v(x) d x\right)^{1 / p}
$$

for every measurable $f$ on $\mathbf{R}^{n}$, if and only if $\sigma\left(B_{Q}\right) \leq c v(Q)^{q / p}$ for every cube $Q$ where $B_{Q}=\{(x, t): x \in Q, 0 \leq t \leq l\}, l$ is the edgelength of $Q$.

See [4] for a proof.

LEMMA (2.2). Let $0<q<\infty$ and $G(x, t)$ be a solution of the heat equation in $\mathbf{R}_{+}^{n+1}$. Then

$$
|G(x, t)|^{q} \leq \frac{C_{q}}{t^{(n+2) / 2}} \int_{\substack{|x-y|<t^{1 / 2} \\ t / 2<s<t}}|G(y, s)|^{q} d y d s .
$$

The last follows by applying Lemma 2 of [2] with $r^{2}=t /(1+n)$.

3. Let $\varphi(t)$ be a nonnegative function on $t>0, \Psi(x)=e^{-\pi|x|^{2}}$ and $F(x, t)=$ $f * \Psi_{t}(x)$. For $u \in D_{\infty}$ and $\varepsilon \in \mathbf{R}$ we define

$$
M_{\varepsilon, u}(f)(x)=\sup _{t>0} \varphi(t) u\left(B_{t}(x)\right)^{\varepsilon / n}|F(x, t)| .
$$

Theorem 1 will be a consequence of the following lemmas.

LEMMA (3.1). Let $0<p \leq q<\infty, \varepsilon>0$, and $u, v \in D_{\infty}$ satisfying (1.2). Then for every $f \in S_{0,0}$ we have

$$
|T f(x)| \leq c\|f\|_{H_{v}^{p}}^{\theta} M_{-\varepsilon, u}(f)(x)^{1-\theta},
$$

where $\theta=\varepsilon /(\varepsilon+n / q)$.

ProOF. For $f \in S_{0,0}$ we have that

$$
T f(x)=2 \int_{0}^{\infty} \varphi(t) F(x, t) \frac{d t}{t} .
$$

In fact, if distance $(\operatorname{supp} \hat{f}, 0)=\lambda>0$ then we have $|F(x, t)| \leq e^{-\pi(t \lambda)^{2}}\|\hat{f}\|_{1}$ and so (3.2) follows by taking Fourier transforms. 
Let $\delta>0$; we write

$$
T f(x)=2\left(\int_{0}^{\delta}+\int_{\delta}^{\infty}\right) \varphi(t) F(x, t) \frac{d t}{t}=I_{1}+I_{2}
$$

Then

$$
\begin{aligned}
\left|I_{1}\right| & \leq 2 \int_{0}^{\delta} \varphi(t) u\left(B_{t}(x)\right)^{-\varepsilon / n} u\left(B_{t}(x)\right)^{\varepsilon / n}|F(x, t)| \frac{d t}{t} \\
& \leq 2 M_{-\varepsilon, u}(f)(x) \int_{0}^{\delta} u\left(B_{t}(x)\right)^{\varepsilon / n} \frac{d t}{t} .
\end{aligned}
$$

Since $|F(x, t)| \leq N(f)(y)$ for $|x-y|<t$ we get that $|F(x, t)| \leq v\left(B_{t}(x)\right)^{-1 / p}\|f\|_{H_{v}^{p}}$ and then by (1.2) we have

$$
\left|I_{2}\right| \leq c\|f\|_{H_{v}^{p}} \int_{\delta}^{\infty} u\left(B_{t}(x)\right)^{-1 / q} \frac{d t}{t} .
$$

Since $u \in D_{\infty}$ implies $u \in R D_{\beta}$ for some $\beta>0$, the lemma then follows by estimating the integrals and minimizing in $\delta$ as in Lemma (3.1) of [5].

LEMMA (3.3). Let $0<p<q<\infty, \varepsilon>0, u \in D_{\mu}$ and $v \in D_{\infty} \cap R D_{\nu}$ with $\nu q>\mu p$. If

$$
\varphi(t) u\left(B_{t}(x)\right)^{1 / q-\varepsilon / n} \leq c v\left(B_{t}(x)\right)^{1 / p}, \quad t>0, x \in \mathbf{R}^{n}
$$

then

$$
\left\|M_{-\varepsilon, u} f\right\|_{q, u} \leq c\|f\|_{H_{v}^{p}} .
$$

ProOF. The function $G$ defined by $G(x, t)=F\left(x,(4 \pi t)^{1 / 2}\right)$ is a solution of the heat equation $\partial / \partial t-\Delta=0$ on $\mathbf{R}_{+}^{n+1}$. Hence by (3.4) and Lemma (2.2) we have

$$
\begin{aligned}
& \left|\varphi(t) u\left(B_{t}(x)\right)^{-\varepsilon / n} F(x, t)\right|^{q} \\
& \quad \leq c v\left(B_{t}(x)\right)^{q / p} u\left(B_{t}(x)\right)^{-1} \frac{1}{t^{n+2}} \int_{\substack{|x-y|<t \\
t / \sqrt{2}<s<t}}|F(y, s)|^{q} s d y d s .
\end{aligned}
$$

Since $v \in D_{\infty}$ we have

$$
M_{-\varepsilon, u}(f)(x)^{q} \leq c \int_{|x-y|<\sqrt{2} s}|F(y, s)|^{q} v\left(B_{s}(x)\right)^{q / p} u\left(B_{s}(x)\right)^{-1} s^{-n-1} d y d s
$$

Integrating this inequality with respect to $u(x) d x$ we get

$$
\left\|M_{-\varepsilon, u} f\right\|_{q, u}^{q} \leq c \int_{\mathbf{R}_{+}^{n+1}}|F(y, s)|^{q} d \sigma(y, s)
$$

where $d \sigma(y, s)=\left\{s^{-n-1} \int_{|x-y|<\sqrt{2} s} v\left(B_{s}(x)\right)^{q / p} u\left(B_{s}(x)\right)^{-1} u(x) d x\right\} d y d s$. We will show that $\sigma$ is a Carleson measure with respect to $v$, i.e.

$$
\int_{0}^{l} \int_{Q} d \sigma(y, s) \leq c v(Q)^{q / p}
$$


for every cube $Q$ with edgelength $l$ and therefore the lemma follows by Lemma (2.1). If we set

$$
I=\int_{0}^{l} v\left(B_{s}(x)\right)^{q / p} u\left(B_{s}(x)\right)^{-1} \frac{d s}{s}
$$

then $I \leq c v\left(B_{l}(x)\right)^{q / p} u\left(B_{l}(x)\right)^{-1}$. In fact, since $u \in D_{\mu}$ and $v \in R D_{\nu}$ we have $u\left(B_{s}(x)\right) \geq c(s / l)^{n \mu} u\left(B_{l}(x)\right)$ and $v\left(B_{s}(x)\right) \leq c(s / l)^{n \nu} v\left(B_{l}(x)\right), 0<s<l$. Hence

$$
I<c l^{-n \nu q / p+n \mu}\left(\int_{0}^{l} s^{-1+n \nu q / p-n \mu} d s\right) v\left(B_{l}(x)\right)^{q / p} u\left(B_{l}(x)\right)^{-1} .
$$

Since

and $\nu q / p-\mu>0,(3.5)$ then follows.

$$
\int_{0}^{l} \int_{Q} d \sigma(y, s) \leq \int_{3 Q} u(x) I d x
$$

PROOF OF THEOREM 1. By Lemma (3.1) we have

$$
\|T f\|_{q, u}^{q} \leq c\|f\|_{H_{v}^{p}}^{\theta q}\left\|M_{-\varepsilon, u}(f)\right\|_{(1-\theta) q, u}^{(1-\theta) q}
$$

with $\theta=\varepsilon /(\varepsilon+n / q)$. For $\varepsilon>0$ small enough we have $(1-\theta) q>p$ and $\nu(1-\theta) q>\mu p$ and since $1 /(1-\theta) q-\varepsilon / n=1 / q$ we may apply Lemma (3.3) and then $\left\|M_{-\varepsilon, u} f\right\|_{(1-\theta) q, u} \leq C\|f\|_{H_{v}^{p}}$.

4. Proof of Theorem 2. We shall first show that

$$
|T f(x)|^{q} \leq c \int_{|x-y|<\sqrt{2} s}|F(y, s)|^{q} s^{-n-1}\left(\int_{s}^{2 s} \varphi(t) \frac{d t}{t}\right)^{q} d y d s .
$$

As in the proof of the Lemma (3.3), by Lemma (2.2) we have

$$
|F(x, t)| \leq c\left(\int_{\substack{|x-y|<t \\ t / \sqrt{2}<s<t}}|F(y, s)|^{q} s^{-n-1} d y d s\right)^{1 / q} .
$$

If $\chi_{B(t, x)}$ is the characteristic function of $B(t, x)=\{(y, s):|x-y|<s, t / \sqrt{2}<s<$ $t$ \} then since $0<q \leq 1$ by (3.2) and Minkowsky's integral inequality we get

$$
|T f(x)|^{q} \leq c \int_{|x-y| \leq \sqrt{2} s}|F(y, s)|^{q} s^{-n-1}\left(\int_{0}^{\infty} \varphi(t) \chi_{B(t, x)}(y, s) \frac{d t}{t}\right)^{q} d y d s .
$$

Since for $|x-y|<\sqrt{2} s$,

$$
\int_{0}^{\infty} \varphi(t) \chi_{B(t, x)}(y, s) \frac{d t}{t} \leq \int_{s}^{2 s} \varphi(t) \frac{d t}{t}
$$

then (4.1) follows. Now integrating (4.1) with respect to $u(x) d x$ we obtain

$$
\|T f\|_{q, u}^{q} \leq c \int_{\mathbf{R}_{+}^{n+1}}|F(y, s)|^{q} \sigma(y, s) d y d s
$$

where

$$
\sigma(y, s)=s^{-n-1}\left(\int_{s}^{2 s} \varphi(t) \frac{d t}{t}\right)^{q}\left(\int_{|x-y|<\sqrt{2} s} u(x) d x\right) .
$$

By the hypothesis made on $u$ and $v$ we have that $\sigma$ is a Carleson measure with respect to $v$ and so by Lemma (1.2) the theorem follows. 


\section{REFERENCES}

1. E. Adams, On the identification of weighted Hardy spaces, Indiana Univ. Math. J. 32 (1983), 477-489.

2. Bui Huy Qui, On Besov, Hardy and Triebel spaces for $0<p \leq 1$, Ark. Mat. 21 (1983), 169-184.

3. S. Chanillo and R. L. Wheeden, $L^{p}$ estimates for fractional integrals and Sobolev inequalities, with applications to Schrödinger operators, preprint.

4. C. L. Fefferman and E. M. Stein, Some maximal inequalities, Amer. J. Math. 93 (1971), 107-115.

5. A. E. Gatto, C. E. Gutiérrez and R. L. Wheeden, Fractional integrals on weighted $H^{p}$ spaces, Trans. Amer. Math. Soc. 289 (1985), 575-589.

6. R. Johnson, Application of Carleson measures to partial differential equations and Fourier multiplier problems, Lecture Notes in Math., vol. 992, Springer-Verlag, Berlin and New York, 1983, pp. 16-72.

7. R. Kerman and E. Sawyer, Weighted norm inequalities for potentials with applications to Schrödinger operators, Fourier transforms and Carleson measures, preprint.

8. J.-O. Strömberg and A. Torchinsky, Weighted Hardy spaces (to appear).

Department of Mathematics, University of Minnesota, Minneapolis, MinNESOTA 55455

Current address: Department of Mathematics, 038-16, Temple University, Philadelphia, Pennsylvania 19122 